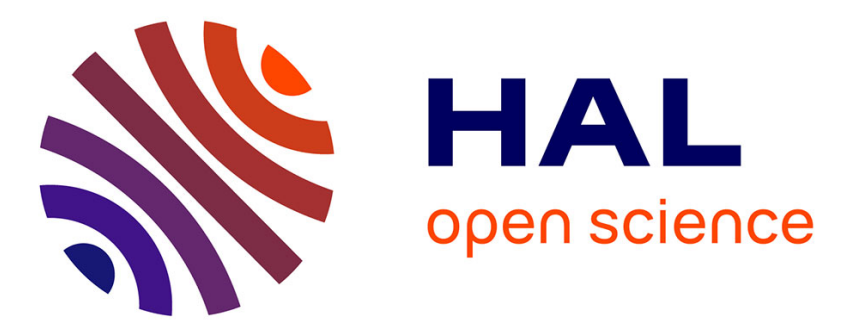

\title{
Phénomènes de co-sélection du lexique et de la grammaire dans le domaine des conjonctions de subordination de certaines langues romanes
}

\author{
Mireille Piot
}

\section{- To cite this version:}

Mireille Piot. Phénomènes de co-sélection du lexique et de la grammaire dans le domaine des conjonctions de subordination de certaines langues romanes. XXVth international Conference on Lexis and Grammar, Sep 2006, Palermo, Italy, France. pp.11. halshs-00307413

\section{HAL Id: halshs-00307413 \\ https://shs.hal.science/halshs-00307413}

Submitted on 29 Jul 2008

HAL is a multi-disciplinary open access archive for the deposit and dissemination of scientific research documents, whether they are published or not. The documents may come from teaching and research institutions in France or abroad, or from public or private research centers.
L'archive ouverte pluridisciplinaire HAL, est destinée au dépôt et à la diffusion de documents scientifiques de niveau recherche, publiés ou non, émanant des établissements d'enseignement et de recherche français ou étrangers, des laboratoires publics ou privés. 


\section{«Phénomènes de co-sélection du lexique et de la grammaire dans le domaine des conjonctions de subordination de certaines langues romanes "}

Mireille Piot

Université Grenoble 3 et LATTICE

\section{Introduction}

Différents phénomènes sont apparus à travers notre étude des conjonctions de subordination (désormais Conjs) du français comme de l'espagnol et de l'italien, mettant en valeur l'étroite interdépendance ou interaction du lexique et de la grammaire dans ce domaine (la grammaire peut être entendue en termes de syntaxe ou de structure, et le lexique en termes de vocabulaire ou d'items sémantiques qui interviennent dans cette structure).

Les phénomènes que nous avons observés sont de deux ordres :

Dans le premier cas (pour lequel nous avons relevé le plus grand nombre d'exemples) un lexique «plein» (non-désémantisé ou non-grammaticalisé) interdit le passage des items correspondants à d'autres structures et donc d'autres classes (ou sous-classes) syntacticosémantiques de conjonctions, alors que, pour la même classe, d'autres items, eux sémantiquement «transparents» (ou grammaticalisés), permettent ces passages. Ces phénomènes concernent différentes classes de nos items dans les trois langues, nous en retiendrons deux exemples ici :

-les passages des «causales »/ « finales» aux «conséquentielles» introduites par par/pour/por/para/per contrairement à pour le motif/ afin/au point (que) et leurs correspondants espagnols et italiens qui ne les permettent pas,

- les « exceptives» : à part/hormis/hors (d'origine 'spatiale') qui peuvent « passer » dans la sous-classe des «additives» (moyennant la perte de contraintes syntactico-sémantiques) contrairement à des items tels que sauf / excepté qui appartiennent à la même sous-classe d' « exceptives » et qui ne permettent pas de tel passage (ni la perte des contraintes corrélées) de même que leurs correspondants espagnols et italiens.

Dans le second ordre de phénomènes (non moins important mais concernant, au moins pour l'instant, moins de classes): les contraintes grammaticales (de parallélisme syntaxique, autrement dit ici de super-structure ${ }^{1}$ ) induisent un comportement particulier amenant à s'interroger sur la nature exacte de l'item en cause par rapport à sa nature habituelle, i.e. dans l'ensemble des autres situations. C'est ainsi que nous avons été amenée à nous interroger, dans des cas précis, sur les items de/de/di constituants de certaines conjonctions de subordination de l'espagnol, du français et de l'italien. En présence de la majorité des conjonctions de subordination ces items sont analysés comme préposition à cause de leur emploi comme introducteur de syntagmes nominaux ou équivalents. Or, des distorsions par rapport au comportement habituel d'une préposition sont constatées pour ces items dans au moins trois classes de conjonctions de subordination : les comparatives, certaines concessives-substitutives, et les additives-exceptives, où interviennent de fortes contraintes de parallélisme syntaxique entre constituants de la principale et de la subordonnée. Nous montrerons que les phénomènes observés invitent à considérer les items de/de/di présents dans ces structures à contraintes de parallélisme comme nettement différents de la préposition de/de/di dont ils partagent cependant les autres propriétés. S'agit-il d'items différents du point de vue de leur nature propre ou bien la nature de la super-structure en cause est-elle responsable de la 'déviance' observée par rapport aux autres cas?

\footnotetext{
${ }^{1}$ Par « super-structure » nous entendons que ces contraintes agissent simultanément et globalement sur l'ensemble formé par les deux phrases conjointes
} 
Ces deux ordres de phénomènes offrent matière à réflexion et des pistes que nous nous proposons ici de présenter et d'approfondir.

\section{Possibilité de passages d'items d'une classe à une autre classe (ou sous-classe) Vs impossibilité.}

Un lexique 'plein' qui comprend notamment des conjonctions formées sur des $\mathrm{N}$ 'appropriés' à certaines notions sémantiques (ou d'autres marqueurs explicites) empêche les passages d'items d'une classe (ou d'une sous-classe) vers une autre alors que des formes plus grammaticalisées (« transparentes », non marquées) le permettent.

\subsection{Ainsi les passages permis entre « causales » / « finales » et « conséquentielles »}

Ce passage est permis pour les Conjs introduites par par/pour/por/para/per mais non autorisés avec pour le motifl afin/au point (que) et leurs correspondants espagnols et italiens; alors que toutes ces Conjs partagent globalement les mêmes propriétés syntactico-sémantiques de leur classe: quelle que soit l'époque (contemporaine ou plus ancienne), pour les trois langues et quelle que soit la nature de la Conjs : Prép SN ( forme non-grammaticalisée) Vs Prép ( forme grammaticalisée). En effet :

- la classe (1) des " causales » est caractérisée par : 1) la nature non-contrainte du sujet de la principale $(\mathrm{Nhum} / \mathrm{N}-\mathrm{hum})$; 2) la subordonnée est à l'indicatif ; 3) lorsqu'elle est réduite à l'infinitif : le temps correspondant peut être le passé comme le montre la possibilité d'insertion de l'adverbe hier (alors que celui du futur, demain, est exclu, à l'inverse de ce qui est observé cidessous avec la classe des « finales »), et cette réduction peut avoir lieu lorsque le sujet de la subordonnée est identique et coréférent à celui de la principale ou à un complément du V principal. Propriétés que montrent les exemples (1a), (2a), et (3a) où figurent des Conjs $(1)=$ Prép de même que dans les exemples (1b), (2b) et (3b) avec les Conjs synonymes de forme Prép Dét $N$ (formées avec les mêmes prépositions et associées à des substantifs «appropriés» comme fait, cause, motif, raison, etc.) :

(1) a Pour(1) (avoir soufflé/souffler) très fort (hier/ *demain), (le vent/ Pierre) a attisé le feu b Pour(1)( la raison/le motif) qu'il a soufflé très fort (hier/*demain), (le vent/ Pierre) a attisé le feu

(2) a Por(1) ser un ladrón (ayer/*mañana), le castigaron

b (Por(1) (el) motivo / por (culpa / razón/ causa) de ser un ladrón (ayer/*mañana), le castigaron

(Pour (le motif/...) d') avoir été un voleur (hier/*demain), on l'a puni)

(3) a Giorgio domandò un' altra volta a Pietro che cosa egli avesse detto, Per(1) non aver sentito bene (ieri/*domani) le sue parole

b Giorgio domandò un' altra volta a Pietro che cosa egli avesse detto, Per(1) (il (fatto/motivo/pretesto)/ la ragione) di non aver sentito bene (ieri/*domani) le sue parole

(Georges demanda une autre fois à Pierre ce qu'il lui avait dit, pour n'avoir pas bien entendu (hier/*demain) ce qu'il disait)

- la classe (2) des "finales » impose les contraintes suivantes : 1) le sujet de la principale est obligatoirement de type " humain », 2) le temps de la subordonnée est obligatoirement le futur comme le montre l'insertion des adverbes aussi bien dans la complétive (que Psubj) que dans la réduction à l'infinitif (même à la forme du passé) qui a lieu obligatoirement 3 ) sous la condition d'identité des sujets de la subordonnée et de la principale; et les $N$ «appropriés» sont ici : but, intention, espoir, dessein, etc. qui forment les autres Conjs de la même classe (avec/dans) le (but/intention/dessein) de.:

(4) a (Pedro/*El viento) sopló fuertemente, (con el objeto de/para) que (sea/*es) avivado el fuego

b (Pierre/*Le vent) a soufflé violemment, (dans le but/pour) que le feu 

(soit/*est) attisé
c (Pietro/*Il vento) soffiò con forza, (affinché/con lo scopo che) (sia/*è) attizzato il fuoco

(5) a (Para/Con la intención de) haberlo acabado (mañana/*ayer), Maria trabaja mucho

b (Pour/Dans le dessein de) l'avoir achevé (*hier / ? en ce moment/ demain), Marie travaille beaucoup

IT c (Per/Nell'intenzione di) averlo finito (domani/*ieri), Maria lavora molto

- - la classe (3) des "conséquentielles » où la Conjs pour(3) associée aux adverbes quantifieurs assez, (in)suffisamment ou trop (peu) peut introduire une complétive au subjonctif (que Psubj), ou sa réduction à l'infinitive $(V$-inf $)$ correspondante, ou bien une complémentation nominale avec une contrainte aspectuelle particulière aux Conjs de cette classe qui s'exerce sur la nature (générique) du Dét=le. La Conjs pour(3) présente les propriétés suivantes:1) comme pour toutes les Conjs de cette classe, la permutabilité en tête de phrase de la séquence Conjs $P$ est exclue, 2) la nature du sujet de la principale est non-restreinte (Nhum ou N-hum), 3) aucune contrainte ne pèse sur le temps de la subordonnée ; ce que montrent les exemples suivants :

(7) Aucun (pays/homme) n'a été suffisamment riche pour payer (hier/demain) la course à l'espace

(8) Ningún (país/hombre) fue lo bastante rico (como) para(2) costear (ayer/mañana) la carrera espacial

(9) La via era troppo stretta perchè potessero passarvi tutti (domani/ieri)

(La rue était trop étroite pour qu'ils puissent y passer tous (hier/demain)

Des $N$ "appropriés" spécifiques de cette classe forment d'autres Conjs de la même classe : point, sorte, façon, manière associés le plus souvent à Dét $=$ tel(le):=à (le/tel) point, de telle (manière/façon/sorte).

Si l'on considère les formes grammaticalisées et notamment les Prép pour/par et leur correspondants romans, la différence entre les trois pour pourrait être interprétée comme relevant d'une image-visée du temps à l'œuvre dans chacun de ces cas. L'antécédence révélée par le temps de la subordonnée ("passé ») dans le cas de pour(1) nous apparaît liée à l'idée de cohérence du discours qui fait qu'un " motif » doit être établi dans un monde réel pour fonctionner comme tel. En revanche, la « finalité » poursuivie (pour(2)) est assurément du domaine du "futur ». Le cas de la subséquence chronologique amorcée par pour(3) semble davantage liée à l'intervention de l'adverbe quantifieur qu'à celle du « temps » de sa subordonnée qui apparaît donc non-contraint.

La différenciation observée entre ces classes dans l'emploi de l'indicatif Vs. subjonctif nous paraît relever, plutôt que de la différenciation 'monde réel / monde possible' souvent évoquée, d'une intervention (prise de participation) de l'énonciateur dans le discours. Ce que montrerait également la possibilité du subjonctif Vs indicatif auprès de certaines Conjs de la classe (1):

(10) Du fait même que Marie est partie (hier/*demain), Pierre est malheureux Du fait même que Marie soit partie (hier/*demain), Pierre est malheureux

En effet, dans ce dernier cas, qu'il s'agisse d'indicatif ou de subjonctif, il s'agit d'un 'motif' établi dans un monde 'réel' et la présence du subjonctif ne change rien aux contraintes observées sur le temps ( "passé » et non «futur» par comparaison avec le temps de la subordonnée « finale ») pour cette classe de Conjs comme le montre l'insertion des adverbes. 
Enfin, le pour de 'cause' (comme le par) permet un renversement de A et de B dans l'ordre linéaire si l'on compare par exemple à l'ordre suivi dans $(12,13)$ contrairement aux Conjs complexes (en $14 \mathrm{c}, \mathrm{d}, \mathrm{e}$ ):

(12) Pour(1) (attiser/avoir attisé) le feu, Pierre a soufflé très fort

(13) Parce qu'il (attise/a attisé) le feu, Pierre a soufflé très fort,

$\neq(14)$ a Pour(1) (avoir soufflé/souffler) très fort (hier/*demain), (le vent/ Pierre) a attisé le feu

b Parce qu'il a soufflé très fort (hier/*demain), (le vent/ Pierre) a attisé le feu

c ?* Pour (le motiflla raison) qu'il (attise/a attisé) le feu, Pierre a dî souffler très for

d ?* Par (le fait/la raison) qu'il (attise/a attisé) le feu, Pierre a dû souffler très fort,

e ?? Du fait qu'il (attisela attisé) le feu, Pierre a dû souffler très fort

(15) a Le castigaron por(1) ser un ladrón (ayer/*mañana)

(ESP)

b Le castigaron por(1) (el) motivo de /por (culpa/razón/causa) de ser un ladrón (ayer/*mañana)

$\neq(16)$ a Es un ladrón por(1) que le castigaron (C'est un voleur parce qu'on l'a puni)

b ?? Es un ladrón, por(1) ((el) motivo / culpa / razón/ causa ) que le castigaron (??C'est un voleur pour le (motif/...) qu'on l'a puni)

(17) a Giorgio domandò un' altra volta a Pietro che cosa egli avesse detto, per(1) non aver sentito bene (ieri/*domani) le sue parole

b Giorgio domandò un' altra volta a Pietro che cosa egli avesse detto, Per(1) (il (fatto /motivo/pretesto)/la ragione) di non aver sentito bene (ieri/*domani) le sue parole

\#(18) a Non aveva sentito bene (ieri/*domani) le sue parole, per(1)ché Giorgio domandò un' altra volta a Pietro che cosa egli avesse detto

(Il n'avait pas bien entendu (hier/...) ce qu'il lui disait, parce que Georges a demandé une autre fois à Pierre ce qu'il lui avait dit)

$\mathrm{b}$ ?? Non aveva sentito bene (ieri/*domani) le sue parole, Per(1) (il (fatto/motivo/ pretesto)/la ragione) che Giorgio domandò un' altra volta a Pietro che cosa egli avesse detto

(?? Il n'avait pas bien entendu (hier/...) ce qu'il lui disait, (pour le fait/le motif/...) que Georges a demandé une autre fois à Pierre ce qu'il lui avait dit)

Ce renversement de $\mathrm{A}$ et $\mathrm{B}$ autour de $\operatorname{pour}(1)$ ou de par entraîne une interprétation où le pour(1) comme le par paraissent viser la 'cause de l'énonciation' plutôt que la 'cause du fait énoncé' : ce qui est le cas univoque de l'autre sous-classe de «causales » qui serait illustrée par puisque dans les mêmes exemples. L'insertion d'un modal auprès de pour(1) ou de par met d'ailleurs en évidence cette interprétation :

(12') Pierre a dû souffler très fort, pour (attiser/avoir attisé) le feu

(13') Pierre a dî souffler très fort, parce qu'il (attise/a attisé) le feu

1.2. Possibilités Vs impossibilités de passage à la sous-classe des «additives » dans le cas des « exceptives» :

Les formes à part/hormis/hors (d'origine spatiale) peuvent «passer» dans la sous-classe des « additives » (moyennant la perte de contraintes syntactico-sémantiques) au contraire d'items tels que sauf / excepté appartenant à la même sous-classe qui ne permettent pas ce passage (ni la perte des contraintes corrélées) de même que leurs correspondants espagnols et italiens.

En effet, appartenant à une même classe syntaxique, les deux sous-classes d' " additives » et d'« exceptives », qui partagent par ailleurs d'autres propriétés, sont nettement différenciées par deux traits qui les opposent :

- Le premier trait correspond à une différence de comportement sur la présence de la négation dans les phrases qu'elles conjoignent. Alors que les « exceptives » imposent un élément négatif 
exprimé ou sous-jacent dans une seule des phrases conjointes (principale ou subordonnée), les « additives » imposent la conjonction de phrases simultanément négatives ou assertives :

(19) (Les enfants/Tous) étai(en)t là, (sauflà part) Pierre,

P1 non-Nég / P2 Nég

$=$ (Les enfants/Tous) étaient là, (sauf/à part) [que] Pierre [n'était pas là],

(Aucun enfant/Personne) n'était là, (sauflà part) Pierre P1 Nég/ P2 non-Nég

$=($ Aucun enfant/Personne) n'était là, sauf [que] Pierre[était là]

(20) a .Outre Marie, (Pierre/les enfants) étai(en)t là

= Outre [que] Marie [était là], (Pierre/les enfants) étai(en)t là

b. Outre Marie, (Pierrelles enfants) n'étai(en)t pas là P1 Nég/P2 Nég

= Outre [que] Marie [n'était pas là], (Pierre/les enfants) n'étai(en)t pas là

- Le second trait concerne la nature du constituant nominal de la principale parallèle à celui 'focalisé' par l'une de ces Conjs :

a) dans le cas des « exceptives », ce constituant doit pouvoir représenter un même 'ensemble' et donc être obligatoirement constitué d'un $N$ 'pluriel' ou 'collectif' ou d'un quantifieur défini, excluant un N'singulier' que permettent au contraire les « additives »:

(19') *Marie était là, sauf Pierre

(20’) Outre Marie, Pierre était là

ensemble auquel appartient comme sous-ensemble, le $N$ de la seconde phrase) :

(21) a Toutes les eaux ont été bues, (sauf/à part/ ...) *le beaujolais

b Toutes les eaux ont été bues, (sauflà part/ ...) la Contrex

b) Au contraire, en présence d'une « focalisation » opérée par outre, le $N$ de la première phrase peut indifféremment être 'singulier', 'pluriel' ou 'collectif', comme le montrent tous les exemples :

(22) a Toutes les eaux ont été bues, (outre/ ...) le beaujolais

b Toutes les eaux ont été bues, (outrel ...) la Contrex

et il s'agit ici d'une union d'ensembles disjoints puisque dans ce cas la Contrex est entendue comme un ensemble différent de toutes les eaux, contrairement aux phrases avec sauf.

Or, nous avons observé avec les plus anciennes des « exceptives » des cas de passage dans la sous-classe opposée des «additives ». Et si l'on considère d'un point de vue morphosémantique, la formation des conjonctions 'additives-exceptives', celle-ci montre le même développement dans toutes les langues romanes que nous avons étudiées :

-les conjonctions les plus anciennes (dès l'origine des langues romanes) 'additives' comme 'exceptives' proviennent d'items (prépositions ou adverbes) originairement 'spatiaux' : (fr.) outre, estre (extra), hors, à part, etc. ;

- les plus tardives (XIVème siècle) des 'exceptives' proviennent de formes adjectivales (ou apparentées) explicites empruntées au langage juridico-administratif: (fr.) sauf, excepté, réservé, etc. . ; situation que nous pouvons résumer par le schéma chronologique suivant :

- (a) Hors/ en dehors de/ hormis/ (mis) à part/: 'espace'(VIe) $\rightarrow$ 'exception'(Xe ) $\rightarrow$ 'addition'

- (b) sauf/ excepté/à l'exception de/... : Adj (VIe ) $\rightarrow$ 'exception'(XIVe) $\rightarrow * * *$ 'addition'

Les plus anciennes des 'exceptives' (grammaticalisées à partir d'items à valeur 'spatiale') peuvent 'glisser' de la sous-classe des 'exceptives' dans la sous-classe des 'additives' moyennant la perte des contraintes sur la négation dans l'une des deux phrases et sur le pluriel ou le quantifieur obligatoire pour le $N$ de la principale comme par exemple dans : 
(23) (A part/Hormis/...) les vins corsés, Pierre aime le whisky $=($ A part/Hormis/...) [le fait qu'il aime] les vins corsés, Pierre aime le whisky]

(24) (A part/Hormis/...) son ordinateur, il est venu avec une mallette = (A part/Hormis/...) [le fait qu'il est venu avec] son ordinateur, il est venu avec une mallette

La 'focalisation' correspond ici à l' 'union' de deux ensembles disjoints (vins corsés et whisky ou ordinateur et mallette), et non à une 'inclusion' (de vins corsés dans whisky, ou d'ordinateur dans mallette); des items tels que à part, hormis, etc. prennent alors la valeur de outre, en plus de, etc.. Ce phénomène de passage d'une sous-classe à l'autre est observable pour tous les états (ancien ou contemporain) de ces langues romanes ainsi par exemple en espagnol :

(25) Son muy densos y suelen estar integrados, aparte de por la sabina negral, por el lentisco $(1998$, CREA)

(Ils sont très denses et sont habituellement intégrés, à part de par le génevrier noirâtre par le pistachier)

(26) Me preguntaron si quería denunciar, aparte de a los delincuentes, a los policías de allí...(2002,Id.

(On me demanda si je voulais accuser, à part de les délinquants, les policiers de là-bas)

Aparte prend ici le sens de además : de toute évidence los delincuentes (les délinquants) ne forment pas un même ensemble avec los policías (les policiers), mais bien des ensembles disjoints.

En revanche, les plus tardives des 'exceptives' comme sauf, excepté, etc. ne permettent pas un tel passage puisque :

(27) *(Sauf/Excepté/...) les vins corsés, Pierre aime le whisky

(28) *Está presente en toda la costa ibérica y balear, a excepción del litoral islandés (2002, remanié)

(*Il est présent sur toute la côte ibérique et des Baléares, à l'exception du littoral islandais)

à cause du caractère 'exceptif' lexicalement explicite alors que les cas précédents (hor $(m i) s, \grave{a}$ part, etc.) partagent avec les 'additives' (outre, etc.) une origine 'spatiale' (signifiant un 'mouvement de passage à partir de...') qui peut convenir à une 'inclusion' ou appartenance à un même ensemble impliquée par exemple par 'hors (de)' ou au contraire à l' 'union' de deux ensembles disjoints ('outre $X, Y$ ').

\section{La nature de $d e / d e / d i^{2}$}

Les phénomènes qui nous intéressent sont observables en présence de certaines classes de Conjs qui imposent de strictes contraintes de parallélisme syntaxique entre phrases (et constituants de ces phrases) qu'elles conjoignent. C'est le cas des comparatives d' "inégalité » de l'espagnol ancien et de l'italien ancien et contemporain, de certaines «concessivessubstitutives » de l'espagnol et des «additives-exceptives» de l'espagnol et du français. En effet, outre le comportement attendu pour une préposition d'introducteur de $S N$, les de/de/di introductrices de ces subordonnées possèdent certaines particularités qui les différencient des prépositions correspondantes : soit elles peuvent introduire d'autres constituants habituellement

\footnotetext{
${ }^{2}$ Problème tout à fait différent de celui du complémenteur de l'infinitif (de/de/di) qui est finalement un simple marqueur (comme que/que/che devant une $\mathrm{P}$ à temps fini) spécifique des propositions infinitives dans l'entourage d'un $\mathrm{V}$ principal et qui est restreint à cette catégorie morpho-syntaxique d'items.
} 
inédits avec une Prép, soit au contraire elles ne peuvent pas introduire de constituants que ces Prép permettent normalement (e.g. Vinf), ou bien enfin elles n'introduisent de $S N$ que dans des conditions très particulières, c'est le cas notamment des comparatives de l'italien ancien.

2.1. Le cas d'introduction de constituants normalement impossibles après une Prép.

\subsubsection{L'introduction d'un syntagme prépositionnel (SPrép ${ }^{3}$ )}

a) Ce cas, possible quelle que soit la Prép simple pour l'espagnol, est abondamment illustré en espagnol ancien pour les comparatives en de (l'espagnol contemporain limitant l'introduction de $S N$ par de aux cas des relatives spécificatives, la même situation ne peut plus être observée) :

(29) No tener gana de rezar ni entretenerse en otra cosa más de en su negocio preciso (CORDE, 1607)

(N'avoir pas envie de prier ni de s'amuser dans autre chose de (=que) dans son commerce...)

(30) Todo lo susodicho no se hace para otra cosa más de para satisfacer al Colegio que lo envía (1552, Id.)

(Tout le susdit ne se fait pas pour autre chose de (=que) pour satisfaire le Collège qui l'envoie)

(31) No lo haces por mal ninguno, mas de por dar algun alivio a tu corazón fatigado.

** 1528 (Id.)

(Ne le fais pour aucun mal, plus de (=que) pour alléger quelque peu ton coeur fatigué)

b) La même situation est également observée en espagnol contemporain en présence des « concessives-substitutives » :

(32) Que se comience por las decenas en lugar de por las unidades (RAE, CREA)

(Que l'on commence par les dizaines au lieu de par les unités)

(33) Con que hubiesen ido por la calzada en vez de por las aceras, 1991 (Id.)

(S'ils étaient allés par la chaussée au lieu de par les trottoirs)

(34) En lugar de en pleno siglo xx lo hubiera hecho en otra época. ** 1991 (Id.)

(Au lieu d'en plein XXème siècle il l'aurait fait en une autre époque)

c) Il en va de même dans le cas des «additives-exceptives » (en présence desquelles interviennent les mêmes contraintes de parallélisme) où de est susceptible d'introduire un SPrép :

(35) Son muy densos y suelen estar integrados, aparte de por la sabina negral, por el lentisco (CREA)

(36) Dichos documentos, además de en castellano, podrán redactarse en dicha lengua. ** (Id.)

(Les dits documents, en plus d'en castillan, pourraient être rédigés dans la dite langue)

(37) Porque afectaba, además de a la mente, al estómago. ** 1993 (Id.)

(Parce qu'il affectait, en plus d'à l'esprit, à l'estomac)

(38) Estos 16 poemas, en los que, a excepción de en los dos últimos, Frost fusiona y funde lirismo 1996(Id.)

(Ces 16 poèmes, dans lesquels, à l'exception de dans les deux derniers, Frost

\footnotetext{
${ }^{3}$ Une suite Prép Prép, possible du point de vue formation morphologique en diachronie, est en revanche synchroniquement totalement exclue en co-occurrence dans nos langues.
} 
fusionne et infuse du lyrisme)

\subsubsection{L'introduction d'un Adj isolé}

Situation représentée pour les « additives-exceptives » de l'espagnol et du français :

(39) a Trabajo que fuera, por otra parte, amén de inútil, peligroso para mí,(RAE, CREA)

b Travail qui serait, d'autre part, en plus d'inutile, dangereux pour moi

(40) a No como crímenes políticos sino puramente racistas, aparte de fascistas 1997 (Id.)

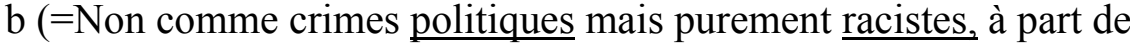
$\underline{\text { fascistes) }}$

(41) a Es, además de estéril, contraproducente ** 1995 (Id.)

b C'est en plus de stérile, contre-productif

(42)Trás de cornudo, apaleado (Id.) = En plus de cocu, battu!

(43) a Alguna laborcica tediosa e inútil, amén de fea $(1881, \mathrm{id})$

b Quelque petite tâche ennuyeuse et inutile, en plus de laide

2.2. Le cas de constituants normalement possibles avec une Prép mais exclus ici.

En effet, l'on observe aussi le cas inverse : la même particule di (de) est, en revanche, exclue dans d'autres structures où la préposition figurerait et serait au contraire normalement attendue. Ce cas se présente dans les « comparatives » en $d i$ de l'italien contemporain qui présentent les propriétés suivantes :

1) contrairement aux comparatives introduites par che (Desideravo partire più che rimanere), elles ne peuvent introduire un infinitif :

(44) *Mi piace più mangiare di bere.(Donati, 2004)[Manger me plaît plus de boire]

et contrairement, également, à la préposition $d i$ complément d'un verbe qui introduit des infinitifs :

(45) Non aveva resistito alla tentazione di riallacciare gli antichi rapporti (Moravia)

(Il n'avait pas résisté à la tentation de renouer les anciens rapports)

2) elles imposent un Dét défini normalement non obligatoire (et même exclu dans les comparatives) auprès d'un $S N$ (Donati, 2004):

(46) Maria mangia più $i$ biscotti delle caramelle=Ddéf Noblig/*Maria mangia più biscotti di caramelle

(Marie mange plus les biscuits de (=que) les bonbons/ Marie mange plus (de) biscuits de (=que) (de) bonbons)

(47) Maria mangia più biscotti che caramelle ( $\left.{ }^{\circ} \mathrm{N}\right)$

(Marie mange plus (de) biscuits que (de) bonbons)

alors même que ce Dét défini n'apparaît pas non plus lorsque la Prép di se trouve en contexte non comparatif :

(48) Maria è capace di parlare per ore di caramelle 
(Marie est capable de parler pendant des heures de bonbons)

\subsection{La Possibilité que le di comparatif introduise en italien ancien un $S N$}

Cette situation est décrite en général et à juste titre comme totalement exclue pour cet état de langue (y compris dans la Grammatica del italiano antico, à paraître), mais elle est exceptionnellement possible. En témoignent les exemples que nous avons relevés chez quelques auteurs toscans du XIIème siècle sous la condition d'un parallélisme strict associé à une opposition sémantique des $S N$ des deux phrases conjointes :

(49) E la figura masculina è più nobele de la feminina, e la figura de l'omo è più nobele de quella de la bestia; adonqua la figura de l'omo, come l'acquario, ch'è più nobele de quella del capricorno, per rascione sarà masculina (Restoro d'Arezzo, 1282 (aret.) L. II, dist. 2, cap.1 70.16 (Gattoweb)

(Et l'image masculine est plus noble de (=que) la féminine, et l'image de l'homme est plus noble de (=que) celle de la bête; ainsi l'image de l'homme, comme le verseau, qui est plus noble de (=que) celle du capricorne, pour cette raison sera masculine)

(50) E valentre e savio, amore d'una donna domanda; e poi viene un altro più valentre $\underline{\mathrm{d} i}$ quello e amore dimanda similemente. Quale sarà da prendere più tosto? Andrea Cappellano volg., a. 1372 (fior.) L. 2 323.21(Id.)

(Et vaillant et sage, amour d'une dame demande ; et puis en vient un autre plus vaillant de (=que) celui-là et amour demande pareillement. Lequel sera à prendre plutôt ?)

Dans tous les exemples traités sous 2., les phénomènes observés invitent à considérer les items $d e / d e / d i$ présents dans ces structures à contraintes de parallélisme comme nettement différents de la préposition de/de/di dont ils partagent cependant les autres propriétés. S'agit-il d'items différents du point de vue de leur nature propre ? Ou bien la nature de la super-structure en cause est-elle responsable de la 'déviance' observée?

Le cas des comparatives des états anciens et/ou contemporains de nos langues romanes pourrait inciter à pencher pour la première hypothèse dans la mesure où $d e / d e / d i$ y apparaît dès l'origine en alternance d'emploi avec le complémenteur que/que/che. Toutefois, les mêmes phénomènes étant susceptibles d'affecter des langues ou des états de langue différents et d'autres classes de Conjs (où de/de/di est d'une autre origine morpho-sémantique), nous optons pour la seconde hypothèse : les fortes contraintes de parallélisme, caractéristique commune de toutes ces classes, nous paraissent constituer un phénomène de super-structure suffisamment puissant pour entraîner les comportements particuliers que nous avons mis ici en évidence.

\section{Conclusion}

Nous avons réuni ici certains phénomènes limités au domaine des conjonctions de subordination de quelques langues romanes qui nous paraissent pouvoir illustrer l'interdépendance entre le lexique et la grammaire. Au travers des exemples cités, nous pensons avoir montré qu'il y a cosélection entre ces deux composantes mais avec, selon les cas, une prédominance de l'une ou de l'autre de ces composantes de la langue. Il est certain que d'autres observations similaires pourraient être apportées dans le même sens sur d'autres secteurs du lexique et/ou de la 
grammaire des langues particulières. Notre observation, limitée pour l'instant aux trois langues romanes sur lesquelles nous avons le plus travaillé, pourrait et devra sans nul doute être étendue aux autres langues romanes et à d'autres langues non apparentées.

\section{Références:}

Donati, Caterina. 2000. La sintassi della comparazione. Padova: Unipress.

Keniston, Hayward. 1930. "Expressions for Than after a comparative in Sixteenth Century Spanish Prose". Revue de Linguistique Romane, 6, p.129-151.

Piot,Mireille. 1995. Composition transformationnelle de phrases par subordination et coordination. Thèse d'Etat

ès Lettres et Sciences Humaines,Université Paris 7 et LADL, 426 p. 1998 .Editions du Septentrion, Coll. « Thèse à la carte ».

Piot, Mireille. 2003. L'expression de la «cause», de la «finalité» et de la «conséquence» : la conjonction pour et ses équivalents en français, espagnol et italien, synchroniediachronie». In (éds),

« La cognition dans le temps », pp. 121-134. Peter Blumenthal ; Jean-Emmanuel Tyvaert

Collection : Linguistische Arbeiten, Tübingen : Niemeyer.

Piot, Mireille. 2005. Sur les fausses prépositions: 'sauf' et 'excepté'. Journal of French Language Studies,

15.3, pp.297-314

Piot, Mireille. 2007. Evolution et fonctionnement des comparatives d'inégalité de l'espagnol, du français et de l'italien (à paraître).

Salvi,Giampaolo; Renzi, Luigi (éds).Grammatica del italiano Antico (à paraître). Bologna: Il Mulino.

Corpus en ligne :

Gattoweb Tesoro della Lingua Italiana delle Origini. Banca dati dell'italiano antico (Corpus OVI dell'Italiano antico),Opera del Vocabolario Italiano, CNR (Italy)

REAL ACADEMIA ESPAÑOLA: Banco de datos (CORDE) [en línea]. Corpus diacrónico del español. $<$ http://www.rae.es> [2005-2006]

REAL ACADEMIA ESPAÑOLA: Banco de datos (CREA) [en línea]. Corpus de referencia del español actual. $<$ http://www.rae.es $>$ [2005-2006]

Adresse de l'auteur:

Mireille Piot

Université Grenoble 3

30, rue Chapon

-F-75003 Paris

France 


\section{Résumé :}

Dans cet article, nous décrivons certains «Phénomènes de co-sélection du lexique et de la grammaire dans le domaine des conjonctions de subordination de certaines langues romanes » qui montrent une étroite interdépendance du lexique et de la grammaire. D'un côté, un lexique plein n'autorise pas certains passages d'items d'une classe à une autre (sous-classe) syntaxiques et inversement un lexique transparent (désémantisé) le permet. D'un autre côté, des comportements d'outils grammaticaux apparaissent déviants, mais, liés à certaines classes sémantiques d'items, ils sont analysables en termes de contraintes de parallélisme syntacticosémantique opérant sur les phrases conjointes.

\footnotetext{
Abstract :

In this paper, we describe some "Phenomena of lexis-grammar co-selection in the Romance subordinating conjunctions domain" pointing out the interaction or very close interdependency of lexis and grammar. On one hand, fully lexical elements do not allow some changes from a syntactic class to another (sub-) class and conversely empty words of the same class allow these changes. On the other hand, some grammatical tool patterns are not in accordance with their original nature, but related to certain semantic classes of subordinating items, and are to be analysed in terms of syntactico-semantic parallelism constraints operating on the clauses under conjunction.
} 